\title{
INVARIANT SETS AND THE HUKUHARA-KNESER PROPERTY FOR SYSTEMS OF PARABOLIC PARTIAL DIFFERENTIAL EQUATIONS
}

\author{
J. W. BEBERNES AND K. SCHMITT*
}

1. Introduction. During the past few years much work has been devoted to the problem of characterizing sets which are invariant with respect to a given ordinary differential equation. More recently the papers [2], [15] have addressed themselves to the same question for nonlinear parabolic differential equations.

The purpose of this paper is twofold. First we provide some extensions of invariance results (for parabolic equations) (sections 3 and 4) and secondly show that the assumptions which are sufficient for a given region to be invariant also yields existence of solutions of initial boundary value problems. We further show that the systems considered have the classical Hukuhara-Kneser property, i.e., the set of solutions of a given initial boundary value problem is a continuum in an appropriate function space; we thus provide an extension of a result of [5] to a large class of systems of parabolic differential equations.

Our invariance results were motivated by a result of $[1]$, where certain geometric conditions were given to establish the solvability of two point boundary value problems for systems of second order ordinary differential equations; the type of result given there is the following: Given a nonempty bounded open convex set such that the vector field defined by the nonlinear terms in the differential equation never points into the interior of the convex set, then for any two points in the convex set there exists a solution of the equation connecting the two points and which has values in that set.

It is precisely these conditions that were adopted in [15] to show that they implied invariance for a system of parabolic equations. Under somewhat weaker assumptions than in [15] we not only prove invariance of that convex region but also demonstrate existence of solutions. Using some ideas suggested by [11] we further show that essentially the same type of result holds for convex sets with empty interior.

In order to establish the Hukuhara-Kneser property for systems of parabolic equations satisfying our conditions we rely on results and ideas about the structure of the set of fixed points of completely con-

*Research supported by U.S. Army research grant DAAG-29-76-G-0186. 
tinuous operators mapping a Banach space into itself which may be approximated by a family of completely continuous operators certain perturbations of which have a (locally) unique fixed point. Such results have a long history (see e.g., [3], [5], [9], [12], [14]), and the version we shall be using is most closely related to that of [3] .

2. Definitions and Notation. Let $R^{n}$ denote $n$-dimensional real Euclidean space and let $\Omega$ be a bounded domain in $R^{n}$ whose boundary $\partial \Omega$ is an $n-1$ dimensional manifold of class $C^{2+\alpha}, \alpha \in(0,1)$. Let $\Pi=\Omega \times(0, \infty), \quad \Pi_{T}=\Omega \times\left(\begin{array}{ll}0, & T\end{array}\right), \quad \Gamma=\partial \Pi, \quad$ and $\quad \Gamma_{T}=(\partial \Omega \times$ $[0, T]) \cup(\Omega \times\{0\})$.

For an arbitrary bounded domain $D \subset R^{n} \times R^{1}$, let $C(\bar{D})$ be the Banach space of continuous functions with domain $\bar{D}$ and range in $R^{n}$ endowed with the usual maximum norm. Let $C^{1,0}(\bar{D})=\{u \in$ $\left.C(\bar{D}): \partial u / \partial x_{i} \in C(\bar{D}), 1 \leqq i \leqq n\right\}$ with norm $|u|_{1}=\max _{x \in D}|u(x, t)|$ $+\max _{x \in \bar{D}, 1 \leqq i \leqq n}\left|\partial u / \partial x_{i}\right|$, and let $C^{\ell, \ell / 2}$ denote the Hölder spaces ([8, page 7$])$, i.e., the set of $u \in C(\bar{D})$ such that $u$ belongs to class $C^{[\ell]}$ ([l] - the greatest integer not exceeding $\ell$ ) with respect to $x$ and the [l] -th partial derivatives of $u$ with respect to the components of $x$ are Hölder continuous with exponent $\ell-[\ell]$ and further $u$ belongs to class $C^{[\ell / 2]}$ with respect to $t$ and $\partial^{[\ell / 2]} u / \partial t^{[\ell / 2]}$ is Hölder continuous with exponent $\ell / 2-[\ell / 2]$. The norms used in these spaces are the Hölder norms (see [8]).

For $u=\left(u_{1}, \cdots, u_{m}\right): \Pi_{T} \rightarrow R^{m}$ define $L u$ by

$$
\begin{aligned}
(L u)(x, t)= & \sum_{i, j=1}^{n} a_{i j}(x, t) \frac{\partial^{2} u}{\partial x_{i} \partial x_{j}}+\sum_{i=1}^{n} b_{i}(x, t) \frac{\partial u}{\partial x_{i}} \\
& +c(x, t) u-\frac{\partial u}{\partial t}
\end{aligned}
$$

where $a_{i j}, b_{i}, c \in C^{\alpha, \alpha / 2}\left(\bar{\Pi}_{T}\right), \alpha \in(0,1), 1 \leqq i, j \leqq n$, and $c(x, t) \leqq 0$ on $\bar{\Pi}_{T}$. We assume that $L$ is a uniformly parabolic operator, i.e., there exist constants $\mu, \lambda, 0<\lambda<\mu$, such that for all $\xi=\left(\xi_{1}, \cdots, \xi_{n}\right)$ and $(x, t) \in \bar{\Pi}_{T}$

$$
\lambda|\xi|^{2} \leqq \sum_{i, j=1}^{n} a_{i j}(x, t) \xi_{i} \xi_{j} \leqq \mu|\xi|^{2}
$$

For $u=\left(u_{1}, \cdots, u_{m}\right) \in R^{m}, p=\left(p_{1}, \cdots, p_{m}\right) \in R^{m n}, p_{i} \in R^{n}, 1 \leqq i$ $\leqq m, f=\left(f_{1}, \cdots, f_{m}\right) \in R^{m}$, let $f: \bar{\Pi}_{T} \times R^{m} \times R^{n m} \rightarrow R^{m},(x, t, u, p)$ $\rightarrow f(x, t, u, p)$ be locally Hölder continuous with Hölder exponents $\alpha, \alpha / 2, \alpha, \alpha$ in the respective variables $x, t, u, p$.

Given $\psi: \Gamma_{T} \rightarrow R^{m}$, consider the initial boundary value problem (IBVP) 


$$
\begin{gathered}
L u=f(x, t, u, \nabla u),(x, t) \in \bar{\Pi}_{T}, \\
u=\psi, \quad(x, t) \in \Gamma_{T},
\end{gathered}
$$

where $\psi$ satisfies compatibility conditions appropriate to the problem, i.e., $\psi$ is continuous on $\Gamma_{T}$ and may be extended to $\bar{\Pi}_{T}$ so as to belong to class $C^{2+\alpha, 1+\alpha / 2}\left(\bar{\Pi}_{T}\right)$.

3. Invariance and Existence. A set $S \subset R^{m}$ is called positively invariant relative to IBVP's for (1) in case given any $\psi$, with $\psi(x, t)$ $\in S,(x, t) \in \Gamma_{T}$, then every solution $u \in C^{2,1}\left(\bar{\Pi}_{T}\right)$ of $(1),(2)$ is such that $u: \bar{\Pi}_{T} \rightarrow S$. A set $S$ is called weakly positively invariant relative to (1) if given any $\psi: \Gamma_{T} \rightarrow S$ there exists at least one solution $u$ of (1), (2) of class $C^{2,1}\left(\bar{\Pi}_{T}\right)$ such that $u: \bar{\Pi}_{T} \rightarrow S$.

TheOREM 1. Let there exist a nonempty, open, bounded, convex neighborhood $S$ of $0 \in R^{m}$ such that for each $u \in \partial S$ there exists an outer normal vector $n(u)$ to $S$ at $u$ such that

$$
n(u) \cdot f(x, t, u, p)>0,
$$

for all $p=\left(p_{1}, \cdots, p_{n}\right), p_{i} \in R^{m}, 1 \leqq i \leqq n$, with $n(u) \cdot p_{i}=0,1 \leqq i \leqq$ $n$, and $(x, t) \in \bar{\Pi}_{T}$. Further let $\psi: \Gamma_{T} \rightarrow S$. Then if $u \in C^{2,1}\left(\bar{\Pi}_{T}\right)$ is a solution of (1), (2), it is the case that $u: \bar{\Pi}_{T} \rightarrow S$, i.e., $S$ is positively invariant.

Proof. Assume not. Then for some $\left(x_{0}, t_{0}\right) \in \Omega \times(0, T), u(x, t) \in S$ for $(x, t) \in \Omega \times\left(0, t_{0}\right)$ and $u_{0}=u\left(x_{0}, t_{0}\right) \in \partial S$. that

By assumption there exists an outer normal $n\left(u_{0}\right)$ to $S$ at $u_{0}$ such

$$
\bar{S} \subseteq\left\{y \in R^{m}:\left(y-u_{0}\right) \cdot n\left(u_{0}\right) \leqq 0\right\}
$$

Put $w(x, t)=\left(u(x, t)-u_{0}\right) \cdot n\left(u_{0}\right)$. Then $w(x, t) \leqq 0$ on $\Omega \times\left[0, t_{0}\right]$ and $w\left(x_{0}, t_{0}\right)=0$. So $w(x, t)$ attains its maximum on $\Omega \times\left[0, t_{0}\right]$ and hence $\left(\Omega\right.$ is open) at $\left(x_{0}, t_{0}\right)$, we have $\partial w / \partial t \geqq 0, \partial w / \partial x_{i}=0$, and $\left(\partial^{2} w / \partial x_{i} \partial x_{j}\right)$ negative semidefinite. We thus conclude that $(L w)\left(x_{0}, t_{0}\right) \leqq 0$. On the other hand, since $\partial w\left(x_{0}, t_{0}\right) / \partial x_{i}=0$ implies $n\left(u_{0}\right) . \partial u\left(x_{0}, t_{0}\right) / \partial x_{i}=0$ we compute

$$
(L w)\left(x_{0}, t_{0}\right)=\left(f\left(x_{0}, t_{0}, u_{0}, \nabla u\left(x_{0}, t_{0}\right)\right) \cdot n\left(u_{0}\right)-c\left(x_{0}, t_{0}\right) u_{0} \cdot n\left(u_{0}\right)>0\right.
$$

(where we have used the fact that $c \leqq 0$ and $u_{0} \cdot n\left(u_{0}\right)>0$ ). This, however, is incompatible with $(L w)\left(x_{0}, t_{0}\right) \leqq 0$. The result thus follows.

Using the preceding invariance result we obtain the following existence theorem. 
THeOREM 2. Let the hypotheses of Theorem 1 hold and let there exist a nondecreasing positive continuous function $\varphi(s)$ satisfying $s^{2} \mid \varphi(s) \rightarrow \infty$ as $s \rightarrow \infty$ and $|f(x, t, u, p)| \leqq \varphi(|p|)$ for $u \in S,(x, t) \in \bar{\Pi}_{T}$. Then for every $\psi: \Gamma_{T} \rightarrow S$ the IBVP (1), (2) has a solution $u: \bar{\Pi}_{T} \rightarrow S$.

Proof. For $u \in C^{1,0}\left(\bar{\Pi}_{T}\right)$ define $F u$ by

$$
(F u)(x, t)=f(x, t, u(x, t), \nabla u(x, t)) .
$$

Then $F: C^{1,0}\left(\bar{\Pi}_{T}\right) \rightarrow C\left(\bar{\Pi}_{T}\right)$ is a continuous mapping taking bounded sets into bounded sets. For $v \in C^{\alpha, \alpha / 2}\left(\bar{\Pi}_{T}\right)$ let $K v$ denote the unique solution in $C^{2+\alpha, 1+\alpha / 2}\left(\bar{\Pi}_{T}\right)$ of the linear problem

$$
\left\{\begin{aligned}
L u & =v, & & (x, t) \in \bar{\Pi}_{T} \\
u & =0, & & (x, t) \in \Gamma_{T},
\end{aligned}\right.
$$

and let (for $\psi$ given) $g$ be the unique solution in $C^{2+\alpha, 1+\alpha / 2}\left(\bar{\Pi}_{T}\right)$ of

$$
\left\{\begin{aligned}
L g=0, & (x, t) \in \bar{\Pi}_{T} \\
g=\psi, & (x, t) \in \Gamma_{T} .
\end{aligned}\right.
$$

Then $K$ is a linear operator and $u=K v+g \in C^{2+\alpha, 1+\alpha / 2}\left(\bar{\Pi}_{T}\right)$ is a solution of

$$
\left\{\begin{aligned}
L u & =v, & & (x, t) \in \bar{\Pi}_{T} \\
u & =\psi, & & (x, t) \in \Gamma_{T} .
\end{aligned}\right.
$$

$K$ may be extended as a bounded linear operator to $L_{q}\left(\bar{\Pi}_{T}\right)$, the extension being again denoted by $K$. Further $\quad K: L_{q}\left(\bar{\Pi}_{T}\right) \rightarrow$ $W_{q}^{2,1}\left(\bar{\Pi}_{T}\right)$ (see [8], p. 343) which for $q$ sufficiently large is embedded in $C^{1+\alpha, \alpha / 2}\left(\bar{\Pi}_{T}\right)$. Hence $K: C^{1,0}\left(\bar{\Pi}_{T}\right) \rightarrow C\left(\bar{\Pi}_{T}\right)$ is a compact linear operator.

For $v \in C^{1,0}\left(\bar{\Pi}_{T}\right), g \in C^{1+\alpha, \alpha / 2}\left(\bar{\Pi}_{T}\right)$ the element $u=K F v+g$ belongs to $C^{1+\alpha, \alpha / 2}\left(\bar{\Pi}_{T}\right)$. Thus if for $u \in C^{1,0}\left(\bar{\Pi}_{T}\right)$

$$
u=\lambda K F u+\lambda g
$$

$\lambda \in[0,1]$, it follows that $u \in C^{1+\alpha, \alpha / 2}\left(\bar{\Pi}_{T}\right)$ and $F u \in C^{\gamma, \gamma / 2}\left(\bar{\Pi}_{T}\right)$ for some $\gamma \in(0,1)$, where in general $\gamma<\alpha$ and $C^{\alpha, \alpha / 2}\left(\bar{\Pi}_{T}\right) \subset C^{\gamma, \gamma / 2}\left(\bar{\Pi}_{T}\right)$; however, if $\gamma \geqq \alpha$, then $F u \in C^{\alpha, \alpha / 2}\left(\bar{\Pi}_{T}\right)$. Thus the IBVP associated with (7) may be considered to have coefficients in $C^{\delta, \delta / 2}\left(\bar{\Pi}_{T}\right)$ where $\delta=\gamma$, if $\gamma<\alpha$ and $\delta=\alpha$ if $\gamma \geqq \alpha$ and the basic existence uniqueness theorem for linear equations guarantees that $u \in C^{2+\delta, 1+\delta / 2}\left(\bar{\Pi}_{T}\right)$.

Summarizing, we have that for any $\lambda \in[0,1]$, if $u \in C^{1,0}\left(\bar{\Pi}_{T}\right)$ is a 
solution of the operator equation (7), then $u \in C^{2+\delta, 1+\delta / 2}\left(\bar{\Pi}_{T}\right)$ and $u$ is a solution of

$$
\left\{\begin{aligned}
L u & =\lambda f(x, t, u, \nabla u),(x, t) \in \bar{\Pi}_{T} \\
u & =\lambda \psi, \quad(x, t) \in \Gamma_{T},
\end{aligned}\right.
$$

and conversely.

If $u \in C^{2+\delta, 1+\delta / 2}\left(\bar{\Pi}_{T}\right)$ is a solution of (8) for some $\lambda$ with $u: \bar{\Pi}_{T} \rightarrow S$, then by the growth condition imposed on $f$ with respect to $\nabla u$, it follows that there exists $M>0$ (independent of $\lambda$ ) (see [8, p. 589]) such that $|\nabla u| \leqq M$.

Let $\mathcal{O}=\left\{u \in C^{1,0}\left(\bar{\Pi}_{T}\right): u: \bar{\Pi}_{T} \rightarrow S,|\nabla u(x, t)|<M+1\right\}$, then $\mathcal{O}$ is a nonempty bounded open subset of $C^{1,0}\left(\bar{\Pi}_{T}\right)$ containing the identically zero function in its interior.

In $C^{1,0}\left(\bar{\Pi}_{T}\right)$, consider the completely continuous perturbation of the identity map

$$
I-\lambda(K F+g), 0 \leqq \lambda \leqq 1 .
$$

The proof will be complete once we show that the Leray-Schauder degree of $I-(K F+g)$ at the point 0 and relative to $\mathcal{O}$ is nonzero, because this property (see [3]) implies that the operator $K F+g$ has a fixed point in $\mathcal{O}$ and thus by the arguments above (1), (2) has a solution $u \in C^{2+\delta, 1+\delta / 2}\left(\bar{\Pi}_{T}\right)$ such that $u: \bar{\Pi}_{T} \rightarrow S$. To verify this claim we use the homotopy-invariance property of Leray-Schauder degree (see [3] ), i.e., we show that the vector field defined by (9) does not vanish on $\partial \mathcal{O}$ for any $\lambda \in[0,1]$. This is clear for $\lambda=0$. If, on the other hand, there exists $\lambda_{0} \in(0,1]$ and $u_{0} \in \partial \mathcal{O}$ such that

$$
u_{0}=\lambda_{0}\left(K F u_{0}+g\right)
$$

then $u_{0}(x, t) \in \bar{S}$ and $|\nabla u(x, t)| \leqq M<M+1, \quad(x, t) \in \bar{\Pi}_{T}$. Thus there exists $\left(x_{0}, t_{0}\right) \in \Omega \times(0, T]$ such that $u_{0}\left(x_{0}, t_{0}\right) \in \partial S$. By Theorem 1 (with $f$ replaced by $\lambda_{0} f$ and $\psi$ by $\lambda_{0} \psi$ ) this is impossible.

Therefore the Leray-Schauder degree

$$
d(I-\lambda(K F+g), 0,0)
$$

is constant for $0 \leqq \lambda \leqq 1$ and hence equal to $d(I, O, 0)=1$. This completes the proof.

4. Some Extensions. In this section we provide some extensions of the result just proved. First we weaken the outer normal requirement and secondly we show that convex sets $S$ with empty interior are also permissible. 
THEOREM 3. Let the hypotheses of Theorem 2 hold with (3) replaced by

$$
n(u) \cdot f(x, t, u, p) \geqq 0 .
$$

Then for every $\psi: \Gamma_{T} \rightarrow \overline{\mathrm{S}}$ the IBVP (1), (2) has a solution $u: \bar{\Pi}_{T} \rightarrow \overline{\mathrm{S}}$.

Proof. For $0<\epsilon \leqq 1$ consider the IBVP

$$
\left\{\begin{aligned}
L u & =f(x, t, u, p)+\epsilon u, & (x, t) & \in \bar{\Pi}_{T} \\
u & =(1-\epsilon) \psi, & (x, t) & \in \Gamma_{T} .
\end{aligned}\right.
$$

It easily follows that Theorem 2 may be applied to conclude that for every $\epsilon \in(0,1]$, (11) has a solution $u_{\epsilon} \in C^{2+\delta, 1+\delta / 2}\left(\bar{\Pi}_{T}\right)$ such that $u_{\epsilon}: \bar{\Pi}_{T} \rightarrow S$ and $\left|\nabla u_{\epsilon}(x, t)\right| \leqq M$, where $M$ is a constant independent of $\epsilon$, not necessarily equal to the constant $M$ of the proof of Theorem 2. Thus $\left\{u_{\epsilon}\right\}_{0<\epsilon \leqq 1}$ is precompact in $C^{1,0}\left(\bar{\Pi}_{T}\right)$.

Since

$$
u_{\epsilon}=K\left(F u_{\epsilon}+\epsilon u_{\epsilon}\right)+(1-\epsilon) g,
$$

the precompactness of $\left\{u_{\epsilon}\right\}$ and the continuity of $K$ and $F$ imply the existence of $u \in C^{1,0}\left(\bar{\Pi}_{T}\right)$ (a limit of a subsequence of $\left.\left\{u_{\epsilon}\right\}\right)$ which satisfies

$$
u=K F u+g
$$

and $u: \bar{\Pi}_{T} \rightarrow \overline{\mathrm{S}},|\nabla u(x, t)| \leqq M$. Hence, as argued before, $u \in$ $C^{2+\delta, 1+\delta / 2}\left(\bar{\Pi}_{T}\right)$ and $u$ is a solution of $(1),(2)$.

REMARK. The condition that $S$ be a neighborhood of 0 may be dropped. This easily follows from the preceding results and a change of variables argument. That $S$ is positively invariant under the conditions of Theorem 3 cannot be concluded. On the other hand $S$ is weakly positively invariant.

We next consider the case where $S$ has possibly empty interior. The idea of the proof was suggested by the results of [11].

THEOREM 4. Let $S$ be a nonempty compact convex subset of $R^{m}$, such that for every $u \in \partial S$ and every outer normal $n(u)$ to $S$ at $u$ we have

$$
n(u) \cdot f(x, t, u, p) \geqq 0,
$$

for $(x, t) \in \bar{\Pi}_{T}$ and those $p$ whose columns $p_{i}$ satisfy $n(u) \cdot p_{i}=0$, $1 \leqq i \leqq n$. Further let there exist a positive, continuous, nondecreasing function $\varphi(s)$ satisfying $s^{2} \mid \varphi(s) \rightarrow \infty$ as $s \rightarrow \infty$ and $|f(x, t, u, p)| \leqq$ $\varphi(|p|), u \in S,(x, t) \in \vec{\Pi}_{T}$.

Then for any $\psi: \Gamma_{T} \rightarrow S$ the IBVP (1), (2) has a solution $u \in$ $C^{2+\delta, 1+\delta / 2}\left(\bar{\Pi}_{T}\right)$ such that $u: \bar{\Pi}_{T} \rightarrow S$. 
Proof. Let $P$ denote the continuous projection $P: R^{m} \rightarrow S$ assigning to each $u \in R^{m}$ its nearest point $P u \in S$, i.e., $|u-P u|=\operatorname{dist}(S, u)$ $=\inf \{|q-u|: q \in S\}$.

Define $h: \bar{\Omega} \times[0, T] \times R^{m} \times R^{m n} \rightarrow R^{m}$ by

$$
h(x, t, u, p)=f(x, t, P u, p) \text {. }
$$

For each $\epsilon>0$, define the parallel set

$$
S_{\epsilon}=\left\{u \in R^{m}: \operatorname{dist}(S, u)<\epsilon\right\} .
$$

Let $u \in \partial S_{\epsilon}$, then $u-P u$ is an outer normal to $S_{\epsilon}$ at $u$ and to $S$ at $P u$. Thus for any $(x, t) \in \bar{\Pi}_{T}, p_{i} \in R^{m}$ with $(u-P u) \cdot p_{i}=0$, we have $(u-P u) \cdot h(x, t, u, p)=(u-P u) \cdot f(x, t, P u, p)$; the latter being $\geqq 0$ by hypothesis. Hence for any $\epsilon>0, h$ satisfies the hypotheses of Theorem 3 relative to the set $S_{\epsilon}$.

Consider the IBVP

$$
\left\{\begin{aligned}
L u & =h(x, t, u, \nabla u), & & (x, t) \in \bar{\Pi}_{T} \\
u & =\psi, & & (x, t) \in \Gamma_{T} .
\end{aligned}\right.
$$

Since $P$ is uniformly Lipschitz continuous with Lipschitz constant $1, h$ satisfies the same smoothness condition as $f$. Hence by Theorem 3 , the IBVP (13) has for every $\epsilon>0$, a solution $u_{\epsilon} \in C^{2+\delta, 1+\delta / 2}\left(\bar{\Pi}_{T}\right)$ such that $u_{\epsilon}: \bar{\Pi}_{T} \rightarrow \bar{S}_{\epsilon}$.

Thus for $0<\epsilon \leqq 1$ the IBVP (13) has a family of solutions $\left\{u_{\epsilon}\right\} \subseteq$ $C^{2+\delta, 1+\delta / 2}\left(\bar{\Pi}_{T}\right)$, with $u_{\epsilon}: \bar{\Pi}_{T} \rightarrow S_{1}$ and there exists $M>0$ (independent of $\epsilon)$ such that $\left|\nabla u_{\epsilon}(x, t)\right| \leqq M, 0<\epsilon \leqq 1$.

As in the proof of Theorem 3 we may extract a subsequence $\left\{u_{\epsilon_{n}}\right\}_{n=1}^{\infty}, \lim _{n \rightarrow \infty} \epsilon_{n}=0$, which converges to a solution $u$ of (13). Since $u_{\epsilon_{n}}: \bar{\Pi}_{T} \rightarrow \bar{S}_{\epsilon_{n},}$ it follows that $u: \bar{\Pi}_{T} \rightarrow S$ and hence $P u(x, t)=$ $u(x, t)$. Thus $u$ is a solution of $(1),(2)$. This completes the proof.

Invariance results similar to those just proved may be obtained for certain sets $\Lambda \subset R^{n} \times R \times R^{m}$ whose cross sections in $R^{m}$ depend on $(x, t) \in R^{n} \times R$. As an illustration of this type result, we shall state a theorem for $\Lambda=\Omega \times(0, T) \times(\alpha, \beta)$, where $(\alpha, \beta) \subset R^{m}$ denotes

$$
(\alpha, \beta)=\left\{u \in R^{m}: \alpha_{i}<u_{i}<\beta_{i}, i=1, \cdots, m\right\}
$$

and $\alpha, \beta \in C^{2+\delta, 1+\delta / 2}\left(\bar{\Pi}_{T}\right)$ satisfy $\alpha_{i}(x, t)<\beta_{i}(x, t)$ for all $(x, t) \in \bar{\Pi}_{T}$. For simplicity in stating the result, we assume that $f$ is gradient independent. The proof of the result is similar to the proof above and hence is omitted. 
TheOREM 5. Assume:

$$
\begin{aligned}
& \left(L \alpha_{i}\right)(x, t)-f_{i}\left(x, t, u_{1}, \cdots, u_{i-1}, \alpha_{i}(x, t), u_{i+1}, \cdots, u_{m}\right) \geqq 0 \geqq \\
& \left(L \beta_{i}\right)(x, t)-f_{i}\left(x, t, u_{1}, \cdots, u_{i-1}, \beta_{i}(x, t), u_{i+1}, \cdots, u_{m}\right), \\
& \quad \text { for }(x, t) \in \bar{\Pi}_{T}, \\
& \alpha_{j}(x, t) \leqq u_{j} \leqq \beta_{j}(x, t), i \neq j, 1 \leqq i \leqq m .
\end{aligned}
$$

Then for any $\psi$ such that

$$
\alpha_{i}(x, t) \leqq \psi_{i}(x, t) \leqq \beta_{i}(x, t),(x, t) \in \Gamma_{T}, 1 \leqq i \leqq m,
$$

the IBVP (1), (2) has a solution $u \in C^{2+\delta, 1+\delta / 2}\left(\bar{\Pi}_{T}\right)$ such that $\alpha_{i}(x, t)$ $\leqq u_{i}(x, t) \leqq \beta_{i}(x, t), 1 \leqq i \leqq m,(x, t) \in \bar{\Pi}_{T}$. If the inequalities in (14) and (15) are strict, then all solutions $u$ of (1), (2) satisfy $\alpha_{i}(x, t)<$ $u_{i}(x, t)<\beta_{i}(x, t), 1 \leqq i \leqq m,(x, t) \in \bar{\Pi}_{T}$.

For results similar to Theorem 5 proving the existence of periodic solutions of parabolic partial differential equations see [13]; other results of this type are contained in [6], [7], [10] .

5. The structure of the solution set. The set of solutions to the initial value problem for ordinary differential equations: $x^{\prime}=f(t, x)$, $x\left(t_{0}\right)=x_{0}$, where $f:\left[t_{0}, t_{0}+a\right] \times R^{n} \rightarrow R^{n}$ is continuous, $a>0$, and $x_{0} \in R^{n}$, satisfies the Hukuhara-Kneser property [4], i.e., if all solutions of the given initial value problem exist on $\left[t_{0}, t_{0}+\delta\right], 0<\delta$ $\leqq a$, then this set of solutions is a continuum in the space of continuous functions defined on $\left[t_{0}, t_{0}+\delta\right]$.

In this section we prove a similar theorem for the set of solutions for the class of parabolic partial differential equations considered above.

This may be accomplished very elegantly by giving an abstracted version of the Hukuhara result for the set of fixed points of completely continuous operators defined in a normed linear space which also satisfy a certain approximation property. Since we need a modification of a known structure theorem [3; p. 89] we include it together with its proof for completeness' sake. Related results may be found in $[5],[9],[12],[14]$.

Theorem 6. Let $X$ be a real normed linear space, $\Omega \subset X$ a nonempty, bounded, open set and $T: \bar{\Omega} \rightarrow X$ a completely continuous mapping. Assume that

$$
d(I-T, \Omega, 0) \neq 0 .
$$


(b) There exists a sequence of completely continuous mappings $T_{n}: \bar{\Omega} \rightarrow X$, such that $\delta_{n}=\sup \left\{\left|T_{n} x-T x\right|: x \in \bar{\Omega}\right\} \rightarrow 0$ as $n \rightarrow \infty$.

(c) $x=T_{n} x+y$, where $y=x_{0}-T_{n} x_{0}, x_{0}$ any solution of $x=T x$, satisfies $|y|<\delta_{n}$, has at most one solution in $\Omega$. $X$.

Then the set of fixed points $Q=\{x \in \Omega: x=T x\}$ is a continuum in

Proof. By assumption (a) the set $Q$ is nonempty and $Q \cap \partial \Omega=\varnothing$. Since $T$ is completely continuous $Q$ is a compact subset of $\Omega$. Assume that $Q$ is not connected. Then there exist nonempty compact subsets $V$ and $W$ of $X$ with $Q=V \cup W$ and $V \cap W=\varnothing$. Since the distances $d(V, W), d(V, \partial \Omega)$, and $d(W, \partial \Omega)$ are positive, there exist open neighborhoods $\Omega_{1}$ and $\Omega_{2}$ of $V$ and $W$, respectively, with $\bar{\Omega}_{1} \cap \bar{\Omega}_{2}$ $=\varnothing$ and $\bar{\Omega}_{1} \cup \bar{\Omega}_{2} \subset \Omega$.

Set $\Omega_{3}=\Omega \backslash\left(\bar{\Omega}_{1} \cup \bar{\Omega}_{2}\right)$ and set $S=I-T$. By the basic properties of Leray-Schauder degree, $d(\mathrm{~S}, \boldsymbol{\Omega}, 0)=d\left(\mathrm{~S}, \Omega_{1}, 0\right)+d\left(\mathrm{~S}, \Omega_{2}, 0\right)$ since $S x \neq 0$ on $\bar{\Omega}_{3}$. Let $\alpha=d\left(0, S\left(\bar{\Omega}_{3}\right)\right)>0$. The proof will be complete once it is shown that $d\left(S, \Omega_{i}, 0\right)=0$, for $i=1,2$, which contradicts the fact that $d(S, \Omega, 0) \neq 0$.

Consider $d\left(S, \Omega_{2}, 0\right)$. Pick $x_{1} \in V$ and define $R_{n}: \bar{\Omega} \rightarrow X$ by $R_{n} x=$ $T_{n} x-T_{n} x_{1}+x_{1}$. Then $R_{n}$ is completely continuous, $R_{n} x_{1}=x_{1}$, and $\left|R_{n} x-T x\right| \leqq 2 \delta_{n}$ for $x \in \bar{\Omega}$. Since $\left|x-R_{n} x\right| \geqq|S x|-\left|R_{n} x-T x\right|$ $\geqq \alpha-2 \delta_{n}$ for $x \in \bar{\Omega}_{3}, d\left(I-R_{n}, \Omega_{2}, 0\right)$ is defined. For $n$ sufficiently large, $x=R_{n} x$ has only the solution $x_{1} \in V \subset \Omega_{1}$ by assumption (c). Thus, $d\left(I-R_{n}, \Omega_{2}, 0\right)=0$.

Let $H: \bar{\Omega}_{2} \times[0,1] \rightarrow X$ be defined by $H(x, t)=(I-T) x+t(T x$ $\left.-R_{n} x\right) . H(\cdot, t)$ is zero-free on $\partial \Omega_{2}$ for all $t \in[0,1]$. For, if not, there exists $x_{0} \in \partial \Omega_{2}, t_{0} \in(0,1)$ such that $H\left(x_{0}, t_{0}\right)=0$. This implies $\left|x_{0}-T x_{0}\right| \leqq t_{0} \cdot 2 \delta_{n}$ which is impossible since $\delta_{n} \rightarrow 0$. Thus, by homotopy invariance of Leray-Schauder degree, it follows that for $n$ sufficiently large $d\left(I-T, \Omega_{2}, 0\right)=d\left(I-R_{n}, \Omega_{2}, 0\right)=0$. Similarly, $d\left(S, \Omega_{1}, 0\right)=0$ and we have thus arrived at a contradiction. We conclude that $Q$ is connected.

The assumption that the approximating equations $x=T_{n} x+y$ have at most one solution for $y$ small where $y=x_{0}-T_{n} x_{0}, x_{0}$ a fixed point for $T$ is a weakening of the condition assumed in [3] and [5]. If one studies the original proof of Hukuhara, it is obvious that our condition (c) is all that was originally used and all that is needed in the abstracted version. Moreover, in the next theorem, the weaker assumption (c) is needed.

If, instead of considering $Q$, the set of fixed points for $T$, we consider $Q_{g}=\{x \in \Omega: x=T x+g, g \in X, T+g: \bar{\Omega} \rightarrow X\}$, the same result holds for $Q_{g}$ provided (c) is replaced by 
(c') $x=T_{n} x+g+\left(T x_{0}-T_{n} x_{0}\right)$ has at most one solution in $\Omega$ for $\left|T x_{0}-T_{n} x_{0}\right|<\delta_{n}, x_{0}$ any solution of $x=T x+g$.

TheOrEM 7. Assume the hypotheses of Theorem 2. Then the set $Q$ of solutions of IBVP (1)-(2) is a continuum in $C^{1,0}\left(\bar{\Pi}_{T}\right)$.

Proof. Using the notation of Theorem 2 and its proof, it suffices to prove that $Q=Q_{\mathrm{g}}=\{u \in \mathcal{O}: u=K F u+g\}$ is a continuum in $C^{1,0}\left(\bar{\Pi}_{T}\right)$ where $\mathcal{O}=\left\{u \in C^{1,0}: u(x, t) \in S,|\nabla u|<M+1, u(x, t)=\right.$ $\psi(x, t)$ on $\left.\Gamma_{T}\right\}$ is as before.

Let $\left\{f_{k}\right\}$ be a sequence of continuous functions on $\bar{\Pi}_{T} \times R^{n} \times R^{m n}$ which are locally Lipschitz continuous with respect to $x, t, u, p$ and converge uniformly to $f$ on compact sets.

For $u \in C^{1,0}\left(\bar{\Pi}_{T}\right)$, define $F_{k}(u)$ by $\left(F_{k} u\right)(x, t)=f_{k}(x, t, u(x, t)$, $\nabla u(x, t))$. Then $F_{k}: C^{1,0}\left(\bar{\Pi}_{T}\right) \rightarrow C\left(\bar{\Pi}_{T}\right)$ is continuous and takes bounded sets into bounded sets for each $k$. Hence, $K F_{k}: C^{1,0}\left(\bar{\Pi}_{T}\right) \rightarrow C^{1,0}\left(\bar{\Pi}_{T}\right)$ is completely continuous for each $k$.

By Theorem 2, $d(I-(K F+g), \quad \mathcal{O}, 0) \neq 0$. By construction, $\sup \left\{\left|K F_{k} u-K F u\right|: u \in \overline{\mathcal{O}}\right\}=\delta_{k} \rightarrow 0$ as $k \rightarrow \infty$. So to apply Theorem 5 (and the remark following it), it suffices to observe that

$$
u=K F_{k} u+g+\left(v-K F_{n} v-g\right)
$$

where $v=K F v+g$ and $\left|K F_{k} v-K F v\right|<\delta_{k}$ has at most one solution. That (16) has at most one solution in $\mathcal{O}$ follows, because for $v \in$ $C^{2+\delta, 1+\delta / 2}\left(\bar{\Pi}_{T}\right)$,

$$
\left\{\begin{aligned}
L u= & f_{k}(x, t, u, \nabla u)+f(x, t, v(x, t), \nabla v(x, t)) \\
& -f_{k}(x, t, v(x, t), \nabla v(x, t)) \\
u(x, t)= & \psi(x, t) \text { on } \Gamma_{T}
\end{aligned}\right.
$$

has at most one solution $u \in \mathcal{O}$. Thus $Q$ is a continuum in $C^{1,0}\left(\bar{\Pi}_{T}\right)$.

A similar argument may be employed to conclude that if strict inequalities hold in the hypotheses of Theorem 5 , then the set of solutions of $(1),(2)$ is a continuum.

\section{REFERENCES}

1. J. Bebernes and K. Schmitt, An existence theorem for periodic boundary value problems for systems of second order differential equations, Arch. Mat. Brno. 8 (1972), 173-176.

2. K. Chueh, C. Conley, and J. Smoller, Positively invariant regions for systems of nonlinear diffusion equations, Ind. Math. J. 26 (1977), 373-392.

3. K. Deimling, Nichtlineare Gleichungen und Abbildungsgrade, SpringerVerlag Berlin: 1974.

4. M. Hukuhara, Sur une généralisation d'un theorème de Kneser, Proceedings Japan Acad. 29 (1953), 154-155. 
5. M. Krasnosel'skii and Sobolevskii, Structure of the set of solutions of an equation of parabolic type, Ukranian. Math. J. 16 (1964), 319-333.

6. W. Mlak, Parabolic differential inequalities and the Chaplighin's method, Ann. Polon. Math. 8 (1960), 139-152.

7. - An example of the equation $u_{t}=u_{x x}+f(x, u)$ with distinct maximum and minimum solutions of a mixed problem, Ann. Polon. Math. 11 (1963), 101-103.

8. O. Ladyzenskaja, V. Solonnikov, and N. Oralceva, Linear and Quasilinear Equations of Parabolic Type, A.M.S. Translations of Mathematical Monographs 23, Providence, 1968.

9. W. Petryshyn, Structure of the fixed points sets of $k$-set-contractions. Arch. Rat. Mech. Anal. 40 (1971), 312-328.

10. G. Prodi, Teoremi di esistenza per equazioni alle derivative parziali non lineari di tipo parabolico, Rend. Inst. Lombardo III (1953), 1-47.

11. K. Schmitt and P. Volkmann, Boundary value problems for second order differential equations in convex subsets of a Banach space, Trans. Amer. Math. Soc. 218 (1976), 397-405.

12. B. Stampacchia, Le trasformazioni funzionali chi presentano fenomeno di Peano, Rend. Accad. Lincei 7 (1949), 80-84.

13. Long-Yi Tsai, Periodic solutions of nonlinear parabolic differential equations, to appear. 42.

14. G. Vidossich, On Peano phenomenon, Bull. Un. Mat. Ital. 3 (1970), 33-

15. H. Weinberger, Invariant sets for weakly coupled parabolic and elliptic systems, Rend. Mat. 8 (1975), 295-310.

University of Colorado, Boulder, Colorado 80302

University of Utah, Salt Lake City, Utah 84112 
, 